\title{
Why Lighting Claims Might Well Be Wrong
}

\author{
Paul Marchant ${ }^{1, *}$ \\ ${ }^{1}$ Visiting Fellow in Statistics, Leeds Beckett University, Leeds, United Kingdom LS1 3HE
}

Received 16 February 2017, Accepted 02 May 2017

\begin{abstract}
This paper gives some background to claims of benefit from road lighting and why large beneficial claims may be suspect. It places this in the context of general concerns about the unreliability of claims in science using knowledge gained, particularly from investigations in the field of health-care.

It points to the need to plan, proceed and check science in unbiased and rigorous ways. It gives recommendations for more transparency, asks for clear protocols to be produced in advance, that clear reports are written which follow appropriate reporting guidelines and that the data is accessible.
\end{abstract}

Keywords: Scientific claims, Evidence, Bias, Statistical analysis, Public safety, Public lighting

\section{Introduction}

In order to know whether any action is sustainable or indeed whether any intervention works, we need good untainted evidence. We need sufficiently full, unbiased, transparent information. We need to know what might be wrong with the evidence presented as well as what is good about it.

The background to this paper is the first part of my oral contribution "Does increasing road lighting increase road injuries?” to ALAN2016, Artificial Light At Night, conference, Cluj-Napoca, Romania in September 2016. I spoke on assessing the effect of changing road lighting in two English cities, to white light, on injury road accidents. The work, to be published, has found nothing like the tens of percent improvement due to new lighting that has been claimed. In fact, the work suggests any reduction in injury accidents, if it exists, is very much smaller; and indeed there is a suggestion of increased harm. It calls into question whether the such a relighting programme represents value for money.

Here I shall go through some issues in the scientific process that may mislead, despite how 'scientific' a claim might appear.

For example, my two batches of comments appended to the Cochrane Systematic Review 'Street lighting for preventing road traffic injuries' [1] highlight some seriously problematic issues with that work. The review, which contained extensive meta-analyses, claimed; "The results from this systematic review suggest that street lighting may prevent road traffic crashes, injuries and fatalities”. The 'Plain Language Summary' and podcasts are more positive about lighting benefit. The review cautioned against the policy, widespread in the UK, of reducing street lighting at night. My critical comments on the other hand said that nothing could be claimed because the review was inherently seriously flawed. Perhaps the points made there explain why the 'LANTERNS project' to determine the effect of reduced street lighting on road casualties and crime in England and Wales, found no detriment from implementing road lighting reductions despite involving a large dataset from 62 local authorities [2].

\footnotetext{
* Marchant P, E-mail address: p.marchant@leedsbeckett.ac.uk
} 


\section{The wider background}

There is currently much concern about dubious and irreproducible claims made in science. Much of this examination and soul searching has taken place in the area of health-care, as in a one page Lancet editorial [3] which outlines problems discussed at a conference on the issue. As an outcome the Academy of Medical Sciences produced a number of documents, of varying length and detail, attempting to address the problems identified [4].

Concerns about the reproducibility and reliability of scientific claims have reached the public media, such as BBC Radio4 [5, 6] and the Economist magazine [7]. In January 2017, the United Kingdom (UK) Parliament, House of Commons Select Committee on Science and Technology launched an inquiry into research integrity to try to address the issue.

\section{Transparency about Potential Conflicts of Interest.}

It is important that full disclosure of potential conflicts of interest is given in any scientific report.

What I see to be my own potential conflicts of interest are:

- The fact I am a lighting sceptic in that I have never seen good evidence that new road lighting enhances public safety from crime or traffic accidents ... (The evidence seen is not robust against a number of threats to scientific validity, which I have written about).

- I have an interest in astronomy and I decided to look into the matter of the claimed benefits of lighting, years ago, because light pollution spoils astronomy.

- However I don’t have financial interests.

I have long been sceptical of claims of lighting benefiting public safety [8-14] so I am clear that I do have an additional potential conflict of interest as it is often painful change one's mind.

However, money would seem to be a major source of bias in research, whether that is through direct financial gain, or through potential gain, for example via a potential job promotion or securing repeat business for providing pleasing results to the paymaster.

The matter of investigating the specific case of lighting, including issues around lack of transparency, is given in [12] \& [15].

\section{Publication Bias}

A huge problem is the fact that not all results are published so that the evidence available is only a subset of all the investigations that were done. This issue of 'publication bias' is known to be still a major problem in the area of health-care. It should be remembered that health-care research is regulated. For example, before a clinical trial takes place a detailed protocol is produced for approval, in advance of collecting any data. It should also be publicly registered so that there is a record of it, which helps to ensure that trials can be followed-up and do not disappear from the scientific record.

The question must therefore arise, "How much bigger is the problem in an unregulated research-area, such as investigating the effects of lighting?” It would seem unlikely that the problem would be smaller and indeed it would seem likely that it might be considerably larger.

The Cochrane Handbook [16] produced by the Cochrane Collaboration, which organises systematic reviews of treatments in health-care, has material on the subject of 'bias'. Bias is the statistical term for assessments homing in on the wrong answer, because of systematic, as opposed to random, errors. The searchable online Handbook is a useful reference for the issue of what makes reliable science.

A major problem is that there is a strong possibility that studies 'go missing' or publishing occurs very late because of their 'uninteresting' or 'unwelcome' findings. Such absence may be associated with the funder. The Handbook gives evidence for this.

That publication bias remains a problem in the regulated world of clinical trials is recognised by the United Nations in the report of the United Nations Secretary-General's High-level Panel on access to Medicines [17]. The recommendations section (4.3.5) concerning clinical trials on page 37 of the report are: 
- Governments should require that the unidentified data on all completed and discontinued clinical trials be made publicly available in an easily searchable public register established and operated by existing mechanisms such as the WHO Clinical Trials Registry Platform, clinicaltrials.gov or in peer reviewed publications, regardless of whether their results are positive, negative, neutral or inconclusive.

- To facilitate open collaboration, reconstruction and reinvestigation of failures, governments should require that study designs and protocols, data sets, test results and anonymity-protected patient data be available to the public in a timely and accessible fashion. Those undertaking clinical trials must not prevent researchers from publishing their findings.

An example highlighting the deficiency is the critical report [18] of the UK House of Commons Public Accounts Committee on its investigation into the transparency and completeness of the evidence on which hundreds of millions of UK-pounds had been spent on an anti-influenza drug.

It was to ensure that full disclosure applies to clinical trials that the AllTrials campaign [19] was set up under the banner 'All Trials Registered, All Results Reported'.

Publication bias seems likely to be large in policy 'trials', especially when something routine like lighting is introduced and there is no registration or protocol in advance. It is not even clear that all such studies are done as experiments. It may only be after implementation that it is decided to prepare a report; once an indication of the result becomes available. A public registry of protocols of planned research would be a valuable contribution to making research on lighting more reliable. Note too that the Cochrane Handbook is critical of Non-Randomised Studies (NRS). These are the kind of studies routinely used to make claims about lighting benefit.

The protocol history for the Beyer and Ker work itself [1] is given on page 56 of the review but there is no mention of there being any protocols of the included studies, despite the critical feedback raising the issue of protocols.

The LANTERNS project [2] has its required protocol available on the website of its funder, the National Institute of Health Research, Public Health Research, See [20]. The full report of the project, Perkins et al [21], is available there for examination also.

\section{Statistical Issues}

It must be remembered that it is not very difficult to get a result out of a piece of statistical software. The difficult thing is to make sure that a result properly addresses a real world problem, and does not arrive at a wrong conclusion and thereby promote a false solution.

In any study through time, there is a need to account for the underlying temporal change, because it is important not to confuse the change which would happen due to the passage of time with that of the effect of the intervention. Therefore an adequate control needs to be used.

When making a comparison of bright road lighting with dim road lighting, it is important to ensure that the comparison made is fair and 'like is being compared with like'. The beauty of the Randomised Control Trial (RCT) is, that because of the random allocation of individual units to the treatments being compared, the treatment groups are statistically equivalent at the start. Therefore, any departure from statistical equivalence detected at the end point of the trial indicates that there has been a difference of effect of treatments.

Beyer and Ker [1] set inclusion criteria for their Cochrane Review. Studies were searched for as described in the review report. The search identified 1308 relevant studies, out of which 17 met the inclusion criteria. These were all controlled before after studies; no RCTs were found. The characteristics of studies are given in the report.

In observational studies, i.e. those without randomisation, there may be important initial differences between the groups being compared which may lead to a wrong conclusion.

Regression towards the Mean (RTM), also known as Regression to the Mean and originally called Regression to Mediocrity when it was first discover by Francis Galton, is one source leading to a biased estimate of effect in studies [22]. It would be wrong to run a 'controlled' before and after study to estimate the effect of increased lighting if this compared areas with a higher crime-rate and given an increase in lighting with other 'control' areas with lower crime rates and unchanging lighting. The biasing effect of RTM increases with the size of starting difference between the groups being compared. It is also greater in a situation where the correlation between the baseline and outcome measurements is weaker and it depends on the form of the bivariate distribution. The likely size of the biasing effect may be estimated [23]. 
It is worth noting that the website 'Understanding Uncertainty' [24] of the team associated with David Spiegelhalter (FRS, Professor of Public Understanding of Risk), independently also criticises work [1] on the basis of RTM and potential publication bias.

It should be remembered that basic statistical theory develops from the assumption that observations of a random variable are independent and identically distributed (IID). That is the observations on a given variable come from the same probability distribution and that each 'realisation' of it is unaffected by any other 'draw'. However, in regard to that last assumption of independence, in the real world what happens before may influence what happens later; that is the observations may be clustered [8]. For a positive association this will increase the variance of an estimate (and is therefore said to be over-dispersed). This is essentially because the nonindependence reduces the effective sample size. The issue of over-dispersion is likely to be influential in studies of crime, as crime is clustered within criminals due to repeat offending, and not important in the occurrence of road collisions as these are more likely to occur in an independent fashion. As stated, criminal behaviour is not independent but clustered, causing higher variability than independence would predict. This is because any criminal may have prolific activity. Therefore, such a person starting and stopping criminal activity will give marked fluctuations in the number of crimes committed. The clustering relates to the fact that the crimes are clustered within each criminal. (The count of crimes in a given area and period will be the sum of all such activity). Contrast this with the situation of repeatedly counting, in a fixed time period, the products of radioactive decay of a piece of long-lived radioactive material. Each radioactive nucleus neither knows nor cares about decaying, it just has the same probability of decaying in each time period. This leads to the counts forming a Poisson distribution. This has defined properties, all characterised simply by its mean.

A further issue is that if the desire is to combine the results of different studies, as in a meta-analysis, it is necessary to ascribe study weights, which determine the magnitude of the contribution that a study gives to the pooled result. These weights are inversely proportional to the variance, which measures the uncertainty around each study's estimate of effect, and the over-dispersion may well be different, and indeed not well-known, in different studies. This will give rise to additional uncertainty in the pooled estimate of effect.

\section{Improving the Registering and Reporting of Studies}

In order to ensure reliable results, it would be desirable to have a registry of work that is planned, to help prevent results of studies disappearing without trace. Having a registry would therefore help improve the completeness of the scientific record.

One of the problems in checking the reproducibility of results is the fact that exactly what occurred in the study can be unclear due to poor and incomplete writing of its report. EQUATOR reporting guidelines [25] exist, in health-care, to encourage report-writing that is clearer and includes all crucial information. The acronym EQUATOR stands for Enhancing the QUAlity and Transparency Of health Research. There are further ideas about improving scientific literature [26].

It would seem to be a good idea to develop reporting guidelines suitable for investigations of the biological impacts of Artificial Light At Night (ALAN), taking into account those already used in health-care. In fact, for impacts of ALAN on humans, use of the health-care guidelines should certainly be encouraged.

Open access to data would also aid transparency and inspire confidence.

\section{Post-Implementation Surveillance.}

There is a need to evaluate what actually has happened after a policy has been implemented. In clinical research this practise is given the name 'Phase IV trial' in which, after a treatment has been approved and it is in routine use, its long-term effects are studied over a lengthy period. By this means rare, important side effects can be picked up, as well as seeing how effective the treatment is in routine use.

In my view there ought to be a requirement to check results as a matter of routine for all public policy implementations. Note the 'roll out' of the policy should be designed so as to make its evaluation, after implementation, straightforward, with the criteria set in advance. The process should be supervised by independent statisticians and scientists. Again unintended effects of the policy might be picked up. As with all science, transparency is important and so open access to data must be required here too. Such checking ought always to be done after large sums of money have been spent, as for example on implementing new road lighting. 
It is important to know whether the money spent has produced the effects in terms of public safety that it was supposed to. Perhaps the large sums of public money spent on road lighting could have been differently and better used in the pursuit of enhancing public safety, without the environmental impact of more artificial light. High quality checking can be done at a small fraction of the cost of the policy implementation.

The LANTERNS project for investigating the effect of lighting reduction was funded, possibly due to a strong belief that road lighting is essential to public safety. Therefore, if the belief is true a reduction in lighting would be expected to have a negative effect on safety; yet no such effect was found.

In contrast to LANTERNS, as yet no project has been set up specifically to evaluate the wide implementation of new road lighting in the UK to see whether it has fulfilled the expectations promised. Such an evaluation might consider the issue of 'value for money' using the evidence it obtained.

\section{Science and Society}

There is a big difference between true science and public relations:

- Science: Get as close as possible to truths about the world. (A balanced process)

- Public Relations: Promote a position in the most favourable way. (A selective process)

It is concerning that academic research seems to be getting more public relations minded.

We need to avoid what Nobel laureate physicist Richard Feynman called 'Cargo Cult Science' [27] by for example trying "to give all of the information to help others to judge the value of your contribution; not just the information that leads to judgment in one particular direction or another".

A cultural shift is needed towards thinking what might reasonably be wrong with research as opposed to blindly accepting the frenzied generation of more research findings of dubious correctness. We need to value the quality of the scientific basis of a study, rather than whether it gives an impressive result which may be erroneous.

There is concern that the current academic system encourages the thriving of bad science at the expense of the good by a process of natural selection [28]. There are some suggestions of how to improve matters [29]. Concerns about dubious claims are not that new [30].

Francis Bacon more than 400 years ago wrote [31] of the practice that those making claims present them in a way that might best believed, and those receiving them are not sufficiently diligent in their scrutiny.

"For as knowledges are now delivered, there is a kind of contract of error between the deliverer and the receiver. For he that delivereth knowledge desireth to deliver it in such form as may be best believed, and not as may be best examined; and he that receiveth knowledge desireth rather present satisfaction than expectant inquiry; and so rather not to doubt, than not to err: glory making the author not to lay open his weakness, and sloth making the disciple not to know his strength.”.

\section{Conclusion}

Much has become clear about the ways that science can produce incorrect answers. There are some safeguards against this problem in place in some areas of study. However, the problems causing erroneous results need to be more generally known. Measures to combat these faults need to be extended to other areas of study, including research on lighting, as outlined above. Doing this should help maintain a good reputation for science and scientists.

The ultimate safeguard against policies that do not do what they are supposed to is that everyone understands and becomes more aware of the issues and is critical of any poor work done; especially that done in the public's name and expense.

\section{References}

[1] Beyer, F. R., \& Ker, K. (2009). Street lighting for preventing road traffic injuries. The Cochrane Library.

[2] Steinbach, R., Perkins, C., Tompson, L., Johnson, S., Armstrong, B., Green, J., Edwards, P. et al. (2015). The effect of reduced street lighting on road casualties and crime in England and Wales: controlled interrupted time series analysis. Journal of epidemiology and community health, jech-2015.

[3] Horton, R. (2015). Offline: What is medicine's 5 sigma. The Lancet, 385(9976), 1380. Available from: http://www.thelancet.com/journals/lancet/article/PIIS0140-6736(15)60696-1/fulltext?rss\%3Dyes. 
[4] Academy of Medical Sciences. Reproducibility and reliability of biomedical research. [Internet]. Acmedsci.ac.uk. 2016 (cited 8 December 2016). Available from: http://www.acmedsci.ac.uk/policy/policyprojects/reproducibility-and-reliability-of-biomedical-research/.

[5] Saving Science from the Scientists - - BBC Radio 4 (cited 8 December 2016). Available from: http://www.bbc.co.uk/programmes/b0742nzq/episodes/player.

[6] Science Betrayed - - BBC Radio 4 (cited 8 December 2016). Available from: http://www.bbc.co.uk/ programmes/b00znb98/episodes/player.

[7] The Economist. Trouble at the lab (cited 8 December 2016); Available from: http://www.economist.com/ news/briefing/21588057-scientists-think-science-self-correcting-alarming-degree-it-not-trouble.

[8] Marchant, P. R. (2004). A demonstration that the claim that brighter lighting reduces crime is unfounded. British Journal of Criminology, 44(3), 441-447.

[9] Marchant, P. (2005). Evaluating area-wide crime-reduction measures. Significance, 2(2), 62-65.

[10] Marchant, P. (2005). Shining a light on evidence-based policy: street lighting and crime.

[11] Marchant, P. (2005). What works? A critical note on the evaluation of crime reduction initiatives. Crime Prevention \& Community Safety, 7(2), 7-13.

[12] Marchant, P. (2006). Investigating whether a crime reduction measure works. Radical Statistics, 91, 41. Available from: http://www.radstats.org.uk/no091/Marchant91.pdf.

[13] Marchant, P. (2010). What is the contribution of street lighting to keeping us safe? An investigation into a policy. Radical Statistics, 102, 32-42. Available from: http://www.radstats.org.uk/no102/Marchant102.pdf.

[14] Marchant, P. R. (2010). Have new street lighting schemes reduced crime in London. Radical Statistics, 104, 32-42. Available from: http://www.radstats.org.uk/no104/Marchant2_104.pdf.

[15] Private Eye. (2005). Conflicts of Interest: Let there be light.1142.28.

[16] Higgins, J. P., \& Green, S. (Eds.). (2011). Cochrane handbook for systematic reviews of interventions (Vol. 4). John Wiley \& Sons. Available from: http://handbook.cochrane.org.

[17] Wilkins, H. (2006). United Nations Secretary-General's High Level Panel on System Wide Coherence and the environment: incremental change or UN reform?. International journal of global environmental issues, 6(4), 285-300. Available from: http://www.politico.eu/wp-content/uploads/2016/09/HLP-Report-FINALSept-2016.pdf.

[18] ACCOUNTS, P. (2013). Access to clinical trial information and the stockpiling of Tamiflu. Available from: http://www.publications.parliament.uk/pa/cm201314/cmselect/cmpubacc/295/29502.htm.

[19] AllTrials (cited 8 December 2016). Available from: http://www.alltrials.net./

[20] Perkins, C., Steinbach, R., Tompson, L., Green, J., Johnson, S., Grundy, C., Edwards, P. et al. (2015). What is the effect of reduced street lighting on crime and road traffic injuries at night? A mixed-methods study. Available from: https://www.journalslibrary.nihr.ac.uk/programmes/phr/11300402/\#/.

[21] Perkins, C., Steinbach, R., Tompson, L., Green, J., Johnson, S., Grundy, C., Edwards, P. et al. (2015). What is the effect of reduced street lighting on crime and road traffic injuries at night? A mixed-methods study. Available from: https://www.journalslibrary.nihr.ac.uk/phr/phr03110\#/full-report.

[22] Marchant, P.R. (2008).Regression towards the Mean. In: Darity WA (ed.) International Encyclopaedia of Social Sciences. 2nd Edition Thomson Gale. 141-143.

[23] Baxter, P.D., Marchant, P.R. (2010). The cross product ratio in the bivariate log normal distribution and gamma distribution, with an application to non-randomised trials, Journal of Applied Statistics, 37(4), 529536.

[24] Understanding Uncertainty. Does street lighting really reduce fatal road crashes by 2/3? (cited 8 December 2016).Available from: http://understandinguncertainty.org/node/231.

[25] Enhancing the QUAlity and Transparency Of health Research (cited 8 December 2016). Available from: www.equator-network.org.

[26] Moher, D., Altman, D.G. (2015) Four Proposals to Help Improve the Medical Research Literature. PLoS Medicine,12(9), e1001864. Available from: http://journals.plos.org/plosmedicine/article?id=10.1371/journal. pmed.1001864.

[27] Feynman, R. Cargo Cult Science: Some remarks on science, pseudoscience, and learning how to not fool yourself. Caltech's 1974 commencement address. (cited 8 December 2016). Available from: http://calteches. library.caltech.edu/51/2/CargoCult.htm.

[28] Smaldino, P. E., \& McElreath, R. (2016). The natural selection of bad science. Royal Society Open Science, 3(9), 160384.

[29] Ioannidis, J. P. (2014). How to make more published research true. PLoS Med, 11(10), e1001747.

[30] Altman, D. G. (1994). The scandal of poor medical research. BMJ: British Medical Journal, 308(6924), 283.

[31] Bacon, F. (1885). The advancement of learning. Clarendon Press. Available from: www.gutenberg.org/files/5500/5500-h/5500-h.htm. 